\title{
Funciones ejecutivas y rendimiento futbolístico. Diseño y evaluación de un programa de intervención \\ Executive functions and football performance. Design and evaluation of an intervention program \\ *Noelia Carbonell Bernal, **María Ángeles Hernández-Prados, *Beatriz R. Sarmiento, *Esther María González Castellón, ***María Cinta Aguaded Gómez, *Julio Alberto Álvarez Núñez \\ *Universidad Internacional de la Rioja (España), **Universidad de Murcia (España), ***Universidad de Nebrija (España)
}

Resumen. Es conocida la relación entre variables neuropsicológicas y destrezas en el ámbito deportivo. Dado que el fútbol es uno de los deportes más practicados en el mundo, es importante analizar el estudio de estas variables en dicho deporte, así como desarrollar intervenciones que contribuyan a mejorar la habilidad deportiva de forma indirecta. La presente investigación tuvo por objetivo averiguar los efectos de un programa de intervención en el que se trabajaron las funciones ejecutivas (FEs) con la finalidad de mejorar el rendimiento en el fútbol. Se llevó a cabo una intervención en la que participaron un total de 29 sujetos de edades comprendidas entre ocho y diez años, que practicaban fútbol de forma habitual. Se realizó una evaluación final para la medición de las funciones ejecutivas, a través del cuestionario para la Evaluación Conductual de la Función Ejecutiva-2 (BRIEF-2). Los resultados apuntan a una relación positiva entre las funciones ejecutiva y el rendimiento en el fútbol.

Palabras clave: Deporte, Funciones Ejecutivas, Fútbol, Infancia, Rendimiento.

Abstract. The relationship between neuropsychological variables and skills in the sports field is known. Taken into account that soccer is one of the most practiced sports in the world, it is important to analyze the study of these variables in this sport, as well as to develop interventions that indirectly contribute to improve sports ability. The present research aimed to find out the effects of an intervention program in which the executive functions were trained in order to improve soccer performance. An intervention program was developed with a total of 29 participants between eight to ten years old, who regularly practice soccer. The executive functions measurement test was carried out through the BRIEF-2 questionnaire. The results point to a positive relationship between executive function and soccer performance.

Key Words: Executive functions, childhood, sport, performance, football.

\section{Introducción y estado de la cuestión}

Afortunadamente el deporte se ha instaurado en la sociedad contemporánea como una práctica cada vez más popularizada en los estilos de vida de la ciudadanía. Se define como una actividad planificada, estructurada y repetitiva, que se realiza con la finalidad de promover la mejora o mantenimiento de ciertos niveles de aptitud y competencia física (Organización Mundial de la Salud (OMS, 2017). El deporte aporta múltiples beneficios en diferentes áreas, tanto para menores como adultos, ya que a nivel psicológico-emocional disminuye el estrés y aumenta la autoestima, y a nivel social mejora la cooperación y las relaciones entre iguales (Bettega, et al., 2018), fomenta la autodisciplina, la resolución no violenta de los conflictos, el rendimiento cognitivo y la regulación emocional (Terry, 2006; Rivas-

Fecha recepción: 29-12-20. Fecha de aceptación: 03-04-21

Beatriz R. Sarmiento

beatriz.rodriguezsarmiento@unir.net
Martínez \& Bailey-Moreno, 2019).

La relación entre las habilidades cognitivas generales y la experiencia deportiva ha sido objeto de interés y análisis, desde hace años, estableciendo que el talento va más allá del dominio deportivo, y requiere del desarrollo de ciertas capacidades cognitivas de índole superior o complejas. Dentro de este enfoque cognitivo, uno de los principales campos en los que se ha centrado el estudio de la neuropsicología deportiva, entendida como la disciplina que se ocupa de estudiar los beneficios y riesgos que entraña la práctica de algún deporte, principalmente desde la dimensión sanitaria y social (Webbe, 2011), ha sido en el de las funciones ejecutivas (FEs), y constituye el primero de los constructos sobre el que se sustenta la presente investigación. Estas funciones vitales para los seres humanos se definen como procesos cognitivos supervisores de la conducta, que implican la organización de acciones de alto nivel cognitivo y ejecutan pensamientos y comportamientos complejos. Se encargan de llevar a cabo una conducta eficaz en entornos tácticos, mediante diversos procesos 
cognitivos que facilitan la anticipación y el establecimiento de metas, la capacidad de autorregularse y de inhibir estímulos que son irrelevantes, transformando el pensamiento en acción (Echavarría 2017; Mikaye, et al., 2000).

Son varios los instrumentos que existen para medir las funciones ejecutivas, poniendo cada uno de ellos el énfasis en unos u otros procesos cognitivos. En este sentido, la adaptación española del cuestionario para la Evaluación Conductual de la Función Ejecutiva-2 (BRIEF2), contempla la planificación, organización de materiales, memoria de trabajo, control emocional, supervisión de sí mismo, supervisión de la tarea, iniciativa, inhibición y flexibilidad cognitiva. Muy similar el Design Fluency Test de Delis, Kaplan y Kramer (2001) se basa en la creatividad, memoria de trabajo, inhibición de respuesta, exploración y flexibilidad cognitiva. Otros autores, como Portellano, Martínez y Zumárraga (2009) miden a partir del cuestionario para la Evaluación Neuropsicológica de las Funciones Ejecutivas en Niños (ENFEN) la planificación, la flexibilidad cognitiva, la memoria de trabajo y la fluidez gráfica, las cuales han sido contempladas también por García-Gómez (2015), añadiendo a su vez, la inhibición y el control emocional. Todo ello evidencia, que las FEs son un constructo multidimensional, integrador de diversos factores y recursos cognitivos de gran valía en el desarrollo comportamental de las personas, que ha despertado el interés científico por conocer y delimitar el papel que desempeñan en diversas esferas vitales, incluida la práctica deportiva. A pesar de su diversidad, se ha acordado en el ámbito de la psicología cognitiva, que la memoria de trabajo, inhibición y flexibilidad cognitiva, constituyen las principales FEs, ya que posibilitan el razonamiento, la resolución de problemas y la planificación (Diamond, 2013).

La relevancia de las FEs en la práctica deportiva ha sido constatada por varios estudios, haciendo referencia cada uno de ellos a diversas modalidades de deporte (baloncesto, tenis, balonmano, boxeo, etc.) y a una o varias funciones ejecutivas, y no tanto al conjunto de ambas como valor global. Así pues, se encuentran diferencias significativas entre jugadores no profesionales, semiprofesionales y de élite en el baloncesto, presentando estos últimos un rendimiento cognitivo superior y más especializado, con una mayor flexibilidad cognitiva y memoria de trabajo, pero sin diferencias en lo referente a la capacidad de inhibición (Alarcón, et al., 2017a). Igualmente, algunas de las FEs están siendo cuestionadas en la práctica deportiva del boxeo, mientras que otras son demandadas. Según el estudio de Ordoqui (2020), este deporte implica un nivel de procesamiento diferente, ya que no solo se opera con una realidad discursiva sino básicamente motora, aconsejando una mayor flexibilidad y creatividad ante las múltiples respuestas posibles que el deportista puede ofrecer ante los estímulos que recibe y los que genera, fruto de sus experiencias previas.

En lo que respecta al fútbol, es innegable que este se encuentra dentro del ranking de los deportes más populares y apreciados en la actualidad, contando con millones de jugadores y admiradores a nivel mundial. Es por estas razones, por las que diferentes disciplinas empíricas han realizado estudios enfocados en el desarrollo y maximización del rendimiento futbolístico; centrándose en la identificación de los factores que tienen un mayor protagonismo en la explicación de la competencia o excelencia individual y colectiva en este deporte, donde se destaca, con gran consenso en la comunidad científica, el factor táctico-estratégico como el más determinante. De hecho, detrás de la práctica del fútbol se localizan múltiples beneficios en los procesos cognitivos relacionados con las funciones ejecutivas, como, por ejemplo, un aumento de la atención, control inhibitorio y memoria de trabajo (Moreno-Luque, et al., 2019).

La identificación, control y puesta en práctica de las funciones ejecutivas por parte de los futbolistas resulta de gran relevancia tal y como se refleja en algunos estudios. Los deportes de equipo, como es el fútbol, requieren tener un buen desarrollo de las funciones ejecutivas que les permita una rápida anticipación y adaptación a situaciones en constante cambio en el campo, la mentalización de posibles opciones de juego, el posicionamiento de los jugadores, el desarrollo de una mayor capacidad visoespacial, una inhibición motora superior y una capacidad mejorada para alcanzar y mantener un estado de alerta, que quienes no hacen deporte, son futbolistas noveles o practican otros deportes (Verburgh, et al., 2014; Vestberg, et al., 2012).

Un jugador de fútbol necesita procesar mucha información en un corto espacio de tiempo y, a menudo, bajo presión mental. Hacer esto de una forma adecuada exige de un elevado dominio de las capacidades cognitivas de orden superior, que a su vez se encuentran condicionadas por las FEs (Beavan, et al., 2019; Zuñiga, et al., 2020). De ahí que en el ámbito futbolístico el deportista que presenta gran capacidad de resolución de problemas, planificación, secuenciación, atención selectiva y sostenida, inhibición, utilización de la retroalimenta- 
ción, la multitarea, la flexibilidad cognitiva y la capacidad para lidiar con la novedad, se encuentra dotado de una mayor inteligencia de juego.

Las FEs no solo constituyen un buen indicador para la selección de talentos deportivos, sino que además son un núcleo de trabajo esencial de los entrenamientos. Por tanto, deben ser consideradas todas las variables que inciden en el aprendizaje-control-dominio de estas, ya sea el tiempo de entrenamiento, variables relacionadas con la relación entrenador-deportista, estados emocionales y condición física de los deportistas. No obstante, aunque se desconocen los factores principales que contribuyen a que las funciones ejecutivas aumenten a lo largo de la carrera de un deportista (Beavan, et al., 2020), las investigaciones parecen concluir que el tiempo de práctica deportiva, ya sea mediante entrenamiento o en los partidos jugados, desempeña un efecto positivo en las FEs, obteniendo mejores puntuaciones, especialmente en la memoria de trabajo, aquellas personas que dedican más tiempo al juego futbolístico (Gómez, et al., 2020; Verburgh et. al., 2016). Otro rasgo que interfiere en el tipo de FEs de los futbolistas es la posición que ocupan en el equipo, de modo que los mediocampistas requieren mayor campo visual y de toma de decisiones, los atacantes más impulsividad y los defensores más funciones de inhibición (Beavan, et al., 2019).

El segundo de los constructos que se abordan en este estudio, el rendimiento deportivo, ha sido definido desde diversas perspectivas. Para Billat (2002) es una acción motriz que permite al individuo expresar potencialidades mentales y físicas durante un entrenamiento, mientras que Navarrete (2019) se refiere a las mejoras que experimentan los deportistas mediante el uso de técnicas para perfeccionar el poder que tiene la mente de concentración y poder rendir adecuadamente, por esos motivos, tiene tanta importancia la salud mental dentro de la actividad física. En definitiva, se trata de la adquisición de distintas competencias, que permiten que el deportista sea calificado de talentoso, y desarrolle la actividad física con una intensidad y eficacia elevada.

Los factores que pueden contribuir en el rendimiento deportivo son: la condición física, estrategia, táctica o la técnica para la condición psicológica, aunque la condición física depende a su vez, de la fuerza, resistencia, velocidad y flexibilidad del individuo. Tras la revisión bibliográfica, se comprueba la tendencia en la investigación deportiva a analizar el papel que juegan las variables psicopedagógicas en la práctica del deporte, mostrando un mayor rendimiento en aquellos depor- tistas que saben gestionar adecuadamente sus recursos psicológicos. De modo que, la motivación, atención-concentración, el grado de ansiedad ante los errores, el nivel de autoconfianza se encuentran condicionados por la percepción de éxito o fracaso en los partidos (González, Campos \& Romero, 2014). Así mismo, el talento deportivo está relacionado con diversos factores, entre los que se encuentran la toma de decisiones, reflexión, control atencional y el adecuado control de las funciones ejecutivas (Serra, Prieto, Pastor y González, 2019). Por tanto, el deportista nace y se hace, pues además de las condiciones físicas, requiere de una serie de competencias como el control de la activación, atención, pensamientos, imágenes mentales, objetivos, conductas y emociones, que se pueden potenciar en los entrenamientos (Palmi \& Riera, 2017) y guardan relación con procesos cognitivos complejos que permiten regular la conducta y emociones.

De igual modo, el rendimiento futbolístico, como deporte de interacción, va asociado a la integración de una serie de habilidades físicas junto a otras de corte psicológico como la planificación, flexibilidad o atención, entre otras (Capuccio, 2017). Siguiendo a Vickers (2007) las habilidades cognitivas que guardan relación con el rendimiento en el fútbol son: concentración, atención, memoria, patrón de reconocimiento, resolución de problemas, toma de decisiones y anticipación, por lo que el trabajo y la mejora de las funciones ejecutivas parece tener una influencia directa en el logro deportivo. Más específicamente, en la evaluación del rendimiento en jugadores de fútbol, realizada por González, Campos y Romero (2014) se han encontrado resultados relacionados con las funciones ejecutivas, de modo que en atención-concentración, más de la mitad de los jugadores no pierde la concentración ante las decisiones arbitrales, ni tampoco ante los comentarios o gestos del entrenador. Finalmente, aunque son muchos los estudios que avalan la relación entre FEs y el rendimiento deportivo, ya que están directamente relacionadas con el éxito en el deporte (Alarcón, et al., 2017a; Hernández-Mendo, et al., 2019), el debate sigue abierto, pues presenta algunas controversias al respecto.

El fútbol en nuestro país, se practica desde edades muy tempranas, fundamentalmente como actividad extraescolar. Este, por sus características, debe considerarse como una actividad con un elevado poder formativo y social, en la que compiten y cooperan, respetan normas y sobre todo, se sienten parte de un equipo. Por lo tanto, si la práctica de este deporte se enfoca de una forma adecuada, se puede conseguir que los alum- 
nos desarrollen conductas positivas, como el respeto, disciplina o la toma de decisiones entre otros elementos (Merino, et al., 2017). De hecho, hay estudios que ponen en relieve los beneficios de practicar fútbol en la etapa escolar en lo que respecta a la mejora de la motivación y el desarrollo de valores como la comunicación o el trabajo colaborativo, entre otros (Macías, GonzálezLópez \& Eslava-Suanes, 2018).

Atendiendo a lo expuesto anteriormente, y en base a las evidencias disponibles, se cree necesaria la investigación que analice los efectos de trabajar de forma sistemática las funciones ejecutivas como parte de la rutina de los entrenamientos en el fútbol con niños entre ocho y 10 años, en el rendimiento deportivo en fútbol. Concretamente, nos planteamos el siguiente problema de investigación: ¿Influye la función ejecutiva en el rendimiento de la práctica del fútbol en niños de edad entre ocho y 10 años? Por ello, la hipótesis de partida planteada fue que existe una relación positiva entre las funciones ejecutivas y el rendimiento en la práctica del fútbol, de manera que cuanto mejor sea la función ejecutiva, mayor será el rendimiento en el fútbol. Para dar respuesta a la cuestión y comprobar si se corrobora la hipótesis inicial, se desarrolló un programa de intervención basado en la observación directa y el análisis de los resultados obtenidos por el equipo durante el campeonato.

\section{Método}

El presente estudio se basa en una metodología correlacional partiendo de un nivel de indagación relacional, que nos permitirá poner de manifiesto los cambios que se producen en le rendimiento futbolístico asociados a los cambios producidos por la innovación en los entrenamientos basada en las FEs. Dicha intervención se lleva a cabo en un solo grupo creado como consecuencia de un muestro no probabilístico, al tratarse de los jugadores de un equipo ya constituido. Ante las carencias observadas por el entrenador en la temporada anterior se plantea la posibilidad de trabajar las FEs de cara a la nueva temporada, recogiendo información durante y después de la intervención. Dicha información consistía en la observación del entrenador así como las narraciones de los participantes del nivel de aplicación de las FEs después de cada partido, y un cuestionario al finalizar la intervención. En definitiva, el objetivo general que planteamos consiste en conocer si las variables objeto de estudio (Índice general de Función Ejecutiva y Rendimiento en el Fútbol), presentan alguna re- lación, o lo que es lo mismo si el rendimiento en la práctica del fútbol en los niños de ocho a 10 años se modifica en relación al entrenamiento en las funciones ejecutivas. Desde aquí, se extraen los siguientes objetivos específicos:

1.Evaluar las funciones ejecutivas y el rendimiento deportivo en una muestra de jugadores de ocho a 10 años.

2.Analizar si existe correlación entre el rendimiento del fútbol y la función ejecutiva.

\section{Participantes}

La población a la que se dirige esta investigación se corresponde con jugadores o jugadoras de fútbol que se encuentren federados o pertenezcan a algún club de fútbol, y cuyas edades abarcan desde los seis hasta los 10 años, pudiéndose adaptar a otros deportes. En consonancia con estas características, se ha extraído una muestra no probabilística por conveniencia compuesta por un total de 29 jugadores federados, todos ellos de género masculino, residentes en Castilla y León (España), de ocho a 10 años de edad, y sin diagnóstico de alteraciones o trastornos de desarrollo neuropsicológico.

Con respecto a las familias de estos niños, se recabó información mediante una entrevista semiestructurada realizada en el momento que realizaron la inscripción al club deportivo. Estas presentan un nivel económico y cultural medio, la mayoría han finalizado estudios primarios, si bien algunos cuentan con titulación universitaria. En el ámbito laboral, el 80\% de las familias trabajan los dos padres, si bien eso no les impide acudir tanto a los entrenamientos como a los partidos, con lo que la implicación de las familias con respecto a este deporte es muy alta, ya que asisten a los entrenamientos y a los partidos de forma habitual, además de colaborar con el equipo en cualquiera de las actividades que se proponen. Para la participación de sus hijos en la presente investigación, los padres cumplimentan un consentimiento informado sobre la misma.

\section{Variables $e$ instrumentos}

Para determinar los niveles de función ejecutiva que presenta la muestra, atendiendo a las edades de los participantes, se seleccionó el cuestionario BRIEF-2 (Evaluación Conductual de la Función Ejecutiva) diseñado para la evaluación de las funciones ejecutivas en niños, niñas y adolescentes de entre cinco y 18 años. Las funciones ejecutivas son definidas como un conjunto de procesos responsables de dirigir, guiar y controlar las funciones cognitivas, emocionales y conductuales, especial- 
mente en aquellos momentos en los que es necesaria una solución de problemas activa por parte de la persona (Santa-Cruz \& Rosas, 2017).

La prueba BRIEF-2 está formada por un cuestionario compuesto por 63 ítems con un formato de respuesta con tres opciones reflejado en una escala tipo Likert (nunca, a veces, frecuentemente), que se cumplimenta en un breve periodo de tiempo (10 minutos aproximadamente). Dispone de dos versiones que comparten la misma estructura: (1) BRIEF-2 Familia, para ser respondida por familiares (padre, madre u otros) y (2) BRIEF-2 Escuela, para ser respondida por profesorado, esta última versión será respondida por los entrenadores.

La presente prueba consta de nueve escalas clínicas (inhibición, supervisión de sí mismo/a, flexibilidad, control emocional, iniciativa, memoria de trabajo, planificación y organización, supervisión de la tarea, organización de materiales). La combinación de estas escalas da lugar a cuatro índices generales: índice de regulación conductual, índice de regulación emocional, índice de regulación cognitiva, índice global de función ejecutiva. Además, incluye tres escalas de validez: infrecuencia, inconsistencia y negatividad. Aspectos altamente relacionados con las variables de estudio, tanto rendimiento en el futbol como funciones ejecutivas.

En la presente intervención la variable FE fue divida en las siguientes subdimensiones en función de las habilidades que las componen: supervisión, inhibición, flexibilidad cognitiva, memoria y planificación.

La administración de esta prueba se realiza de forma individual y sus resultados equiparan las puntuaciones altas con la presencia de problemas en el área que representa la escala en la que se obtenga.

En lo que respecta a la variable rendimiento deportivo en fútbol, este término se define cómo la consecución de los objetivos establecidos. Para evaluar esta variable, se utilizaron las observaciones registradas por parte del entrenador de cada uno de los jugadores al finalizar el entrenamiento o el partido, pues tal y como expone Costa, Camerino y Sequeira (2015) se trata de una metodología idónea para contextos naturales, exentos de posibles manipulaciones. Entre los ítems a observar se especifica el número de pases acertados, el número de pases errados, bloqueos de balón, colocación en el campo y número de goles y la clasificación general del equipo, a la hora de realizar la valoración. Estos aspectos nos delimitarán las variables a medir para analizar si existe correlación entre la función ejecutiva y el rendimiento en el fútbol. Se evalúan estos ámbitos por- que están estrechamente relacionadas con las variables de estudio.

\section{Procedimiento y programa de intervención}

Con la intención de cubrir los parámetros éticos de la investigación y las indicaciones contempladas en las normas APA de ética en la investigación (American Psychology Association, 2017), se mantuvo una reunión con la junta directiva del club para exponer el proyecto de investigación a desarrollar con los niños del club y una vez obtenida su aprobación, se convocó a las familias de los jugadores para informarles del proyecto y solicitarles su consentimiento firmado, ya que los destinatarios de la intervención son menores de edad. Allí mismo se citó a las familias para que asumieran un papel colaborativo importante, consistente en mantener continuidad y puntualidad en las sesiones de entrenamientos y en los partidos, asistiendo de forma regular y evitando faltar, salvo excepciones justificadas.

Con la autorización de las familias, se inició el programa de intervención para la mejora del rendimiento deportivo a partir del desarrollo de la función ejecutiva, con una temporalización de dos sesiones por semana durante ocho meses, lo que computa un total de 64 sesiones, que oscilan de cuatro a 10 sesiones dependiendo de los festivos que se acumulen en un mismo mes. De modo que, en 2018, año de aplicación del programa, septiembre, diciembre y marzo son los meses con menos entrenamientos, y octubre el que más (véase Tabla 1). Se siguió una metodología multisensorial, activa, dinámica y participativa, centrada fundamentalmente en el rendimiento individual de cada niño.

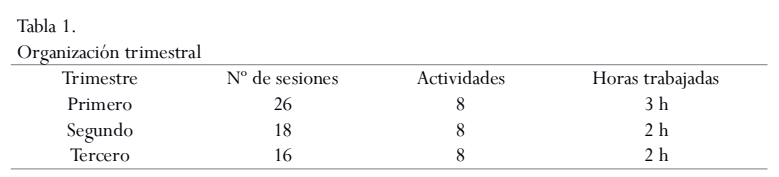

La constancia en las actividades rutinarias que el entrenador marcó a los participantes resulta esencial para poder aumentar los niveles de motivación en los jugadores, así como su rendimiento deportivo en los partidos. Con este propósito, en cada sesión de entrenamiento, concretamente dos días a la semana durante una hora y cuarto, se destinaban los primeros minutos a la realización de una actividad con los alumnos sobre algunas de las subdimensiones de la función ejecutiva y no de forma global o general, ya que de esta forma el trabajo era más exhaustivo, así como el respectivo seguimiento y toma de datos continua de cada jugador. Las actividades a trabajar iban en función del contrincante con 
el que se jugaba ese fin de semana. El volumen de horas de trabajo en las FEs en el Club de Fútbol asciende a unas 20 en total, las cuales se ven complementadas por la repetición de estas mismas tareas en el contexto familiar bajo la supervisión de los padres. El resto de la sesión de entrenamiento se dedicaba al rendimiento deportivo en fútbol.

El fútbol es un deporte aeróbico que requiere coordinación y en el que para la obtención de beneficios, es necesario ir mejorando las habilidades paulatinamente. Partiendo desde esta idea, se explicó a los niños cada una de las tareas que iban a trabajar, incidiendo de forma particular en que la práctica les llevaría a la mejora en el rendimiento futbolístico. Así, se muestra en qué va a consistir la nueva rutina con la que comenzarán los entrenamientos, introduciendo el término funciones ejecutivas, para que se familiaricen con el mismo y conozcan de primera mano la importancia del adecuado manejo de estas en la vida diaria y en el deporte.

Por lo tanto, a lo largo de la temporada se desarrollaron unas actividades concretas centradas en el trabajo de las funciones ejecutivas (control inhibitorio, flexibilidad cognitiva, supervisión y planificación y memoria) a través de la participación individual y grupal, buscando la estabilidad entre la motivación y la creación sistemática de rutinas. Todas las tareas que se van a realizar fueron descritas antes del comienzo de la sesión con la finalidad de que ellos puedan comprender cuál es la objetivo del ejercicio que van a efectuar, y deben ser realizadas en el campo de entrenamiento. Además de las actividades mencionadas, se realizaron varias reuniones con las familias en las que mediante un grupo de discusión se recogieron las percepciones que tenían sobre el programa y los cambios que habían promovido en sus hijos, como por ejemplo si notaban cambios comportamentales en sus hijos, las notas académicas, su forma de jugar al fútbol, la actitud que muestra antes y después de un entrenamiento o de un partido y el dialogo que establecen con ellos. Por otro lado, después de cada sesión y partido, se les pidió a los jugadores que llevarán a cabo la evaluación de la misma.

Finalizado el programa, se aplicó el cuestionario BRIEF-2 a la muestra de 29 sujetos en el día y hora acordados para cada uno de ellos. Se utilizó dicha prueba dado que se adaptaba al objetivo de este estudio y es una prueba normalmente usada por investigadores en estudios e investigaciones de temáticas similares. A continuación, se realizó el análisis de los resultados obtenidos y la evaluación del rendimiento en el fútbol del grupo de alumnos.

A continuación, se recogen descriptivamente las actividades que se han desarrollado durante el entrenamiento (Véase Tabla 2). Existen ocho modalidades diferentes de actividades que trabajan distintas subdimensiones de la función ejecutiva. En cada sesión de entrenamiento se aplica una de ellas de forma sistemática y secuencialmente, de modo que al finalizar todas se inicia nuevamente la secuencia.

Se debe tener en cuenta que el entrenador es el que dirige las actividades y realiza las anotaciones oportu-

Tabla 2.

\begin{tabular}{|c|c|c|}
\hline Actividad & Subdimensión FE & Desarrollo \\
\hline Atentos a la orden & $\begin{array}{l}\text { - Supervisión } \\
\text { - Planificación }\end{array}$ & $\begin{array}{l}\text { Todos los alumnos deben colocarse en círculo alrededor del entrenador y comenzar a trotar estableciendo una separación de cinco metros. Cuando el entrenador } \\
\text { dice "ya", todos deben correr hacia él y volver a la posición original. }\end{array}$ \\
\hline Gymkana & $\begin{array}{l}\text { - Supervisión } \\
\text { - Inhibición } \\
\text { - Flexibilidad cognitiva } \\
\text { - Planificación }\end{array}$ & $\begin{array}{l}\text { Circuito formado por un total de siete pruebas, en el siguiente orden: } \\
\text { - elevación de rodillas } \\
\text { - elevación de talones } \\
\text { - carrera de zigzag entre conos } \\
\text { - carrera con el balón en zigzag según los conos marcados } \\
\text { - salto de aros y picas } \\
\text { - conducción del balón en velocidad } \\
\text { - trabajo en una portería pequeña }\end{array}$ \\
\hline $\begin{array}{l}\text { Seguimos un } \\
\text { orden }\end{array}$ & $\begin{array}{l}\text { - Supervisión } \\
\text { - Inhibición } \\
\text { - Planificación }\end{array}$ & $\begin{array}{l}\text { La actividad comienza poniendo a los niños en su posición en el campo (pondremos un elemento al lado como punto referente). Se marcan tres zonas del campo } \\
\text { con diferentes colores (verde, rojo y azul) y los niños deberán ir a la zona que les indiquen, pero sin perder su colocación en el campo. }\end{array}$ \\
\hline Tres en raya & $\begin{array}{l}\text { - Atención } \\
\text { - Flexibilidad cognitiva }\end{array}$ & $\begin{array}{l}\text { El equipo es dividido en dos grupos de dos colores con tres petos en los tres primeros de cada fila. Acto seguido se colocan cuatro conos para que tengan que correr } \\
\text { antes de llegar a la zona de tres en raya. La actividad consiste en salir corriendo un jugador de cada fila con el peto en la mano, hacer el circuito de las cuatro picas y } \\
\text { llegar a la zona de tres en raya con el peto del color y posarlo en un aro, volver a la zona de la fila corriendo y chocarle la mano al siguiente para que pueda el } \\
\text { realizar lo mismo que el anterior, aś hasta que un equipo haga tres en raya y gane. Si se da la casualidad de que están los tres petos de cada equipo en la zona de tres } \\
\text { en raya pero aún no ha salido un equipo ganador los jugadores seguirán haciendo el circuito hasta que un equipo consiga hacer tres en raya. }\end{array}$ \\
\hline $\begin{array}{l}\text { Control de la } \\
\text { pelota }\end{array}$ & $\begin{array}{l}\text { - Supervisión } \\
\text { - Planificación } \\
\text { - Memoria }\end{array}$ & $\begin{array}{l}\text { Se divide a los niños en parejas y a cada grupo se le da una pelota, a una distancia de siete metros, el entrenador va marcando como se realizan pases, primero se } \\
\text { realizan pases con control y pase con la misma pierna, a continuación indica control con una pierna y pase con la otra pierna, a continuación ordena realizar pases de } \\
\text { primeras sin control, a continuación ordena antes de dar el pase mirar hacia atrás (por si hubiese un contrario en situación de partido), todos ellos para conseguir un } \\
\text { buen dominio del balón }\end{array}$ \\
\hline $\begin{array}{l}\text { Actividades con } \\
\text { colores }\end{array}$ & $\begin{array}{l}\text { - Supervisión } \\
\text { - Memoria } \\
\text { - Flexibilidad cognitiva } \\
\text { - Planificación }\end{array}$ & La actividad consiste en realizar por parejas ejercicios según el color que diga el entrenador (véase Figura 1). \\
\hline $\begin{array}{l}\text { El juego del } \\
\text { pañuerlo }\end{array}$ & $\begin{array}{l}\text { - Supervisión } \\
\text { - Memoria } \\
\text { - Planificación }\end{array}$ & $\begin{array}{l}\text { El entrenador debe realizar dos equipos, delimitar la zona del pañuelo y la zona de cada equipo (con conos). La actividad consiste en que un jugador toma el pañuelo } \\
\text { en la zona delimitada y deberá decir números, según el número que diga, saldrá corriendo el jugador que lo tenga asignado hacia el pañuelo, intentando cogerlo y } \\
\text { que el compañero del otro equipo no le pille. Así hasta que solo quede uno de cada equipo o un equipo se quede sin jugadores }\end{array}$ \\
\hline $\begin{array}{l}\text { Atrápame si } \\
\text { puedes }\end{array}$ & $\begin{array}{l}\text { - Memoria } \\
\text { - Flexibilidad cognitiva }\end{array}$ & $\begin{array}{l}\text { La actividad consiste en atrapar, al contrario, el entrenador hace parejas, marca una línea sobre el campo y otra a una distancia de siete metros a cada lado de la línea } \\
\text { del centro. Coloca uno de la pareja de cada lado de la línea. A los que están del lado derecho de la línea les asigna "números pares" y a los del lado izquierdo } \\
\text { "números impares". El entrenador debe decir un número, par o impar y según el número que diga el jugador deberá correr hasta la línea que está a siete metros, } \\
\text { para no ser pillado. }\end{array}$ \\
\hline
\end{tabular}



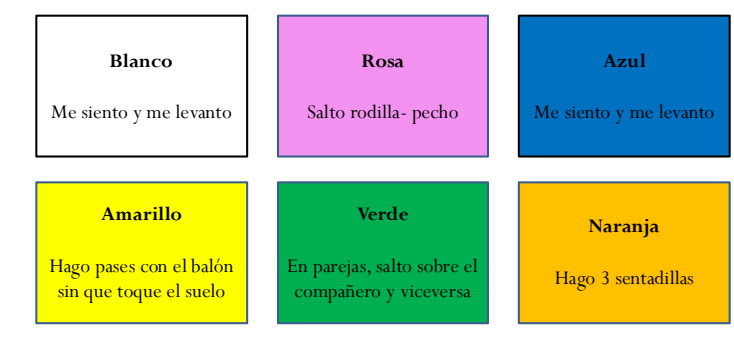

Figura 1. Colores e instrucciones.
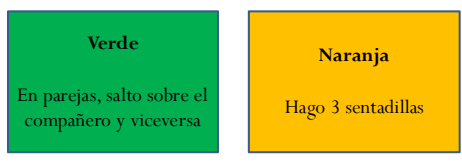

nas de cada jugador. En todas ellas, los jugadores deben estar atentos, seleccionar la información que es relevante de la que no lo es, mantener el control inhibitorio, la capacidad de anticiparse y pensar para poder realizar la acción, controlar sus reacciones y emociones, habilidad para producir respuestas verbales, decidir rápidamente otra respuesta y poder elegir entre varias alternativas.

\section{Resultados cuantitativos y cualitativos}

Dada la naturaleza del estudio se han recabado datos de índole cuantitativo (BRIEF-2 y observación sistemática) y cualitativo (narraciones). A continuación se describen los resultados.

En primer lugar, se muestran los descriptivos de ambas variables, reflejados como los valores de media y desviación típica obtenidos en el rendimiento en el fútbol $(M=6,7 ; D T=1,29)$ y en la función ejecutiva $(M=6,16 ; D T=1,48)$.

Atendiendo a los valores obtenidos en el rendimiento deportivo a través de la observación participante del entrenador, del total de 29 niños 14 presentan un rendimiento deportivo bueno (46.66\%), en el nivel medio o regular encontramos a 10 alumnos, que representan un $30 \%$ de la muestra, y en el peor nivel del rendimiento deportivo nos encontramos con 5 niños, siendo el porcentaje del $23.44 \%$. Respecto a las funciones ejecutivas, medidas en una escala de 0 a 10 con el cuestionario BRIEF-2 tras la aplicación del programa de intervención, obtenemos los siguientes resultados: la mayoría de los alumnos se sitúan entre los valores 5 y 7 , repartidos de la siguiente manera $27.5 \%$ (8 niños) se sitúan en el valor $5,17,2 \%$ corresponde a los 5 niños que se sitúan en el valor 6 y en el valor 7 se encuentran un $24.1 \%$ (7 alumnos).

Tal y como se aprecia en la Figura 2, podemos ver cuál es la relación entre estas variables, viendo como los niveles de funciones ejecutivas y el rendimiento futbolístico son elevados tras la realización de las actividades planteadas en el programa y lo que es más impor- tante presentando valores similares en la puntuación. Los análisis estadísticos muestran una correlación positiva entre ambas variables, de manera que cuanto mejor es el rendimiento en la práctica del fútbol, mejor es la puntuación en función ejecutiva $(r=.871)$.

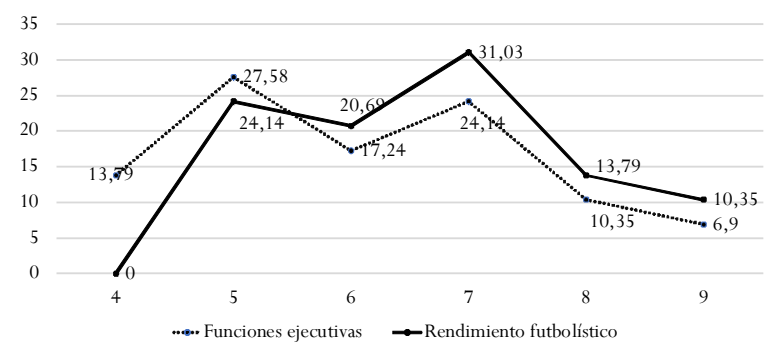

Figura 2. Relación entre el rendimiento futbolístico y las funciones ejecutivas.

En definitiva, estos resultados apuntan a una relación entre el nivel de dominio de las funciones ejecutivas y el índice de rendimiento futbolístico.

Con respecto a las reuniones con las familias y los datos por ellas suministrados recogimos que la respuesta fue positiva, pues al finalizar el programa de intervención reconocían que se habían producido varias mejoras: el aumento del rendimiento escolar y deportivo, una mayor implicación en las actividades realizadas, parecían más concentrados y salían muy satisfechos de los entrenamientos y los partidos.

En función de las evaluaciones de los jugadores se puso de manifiesto como al inicio de la intervención, les era más difícil concentrarse y prestar atención a las actividades, ya que suponían el empleo de las funciones ejecutivas y los jugadores aún no estaban familiarizados con la utilización de estas, por otro lado, no debemos olvidar que el rango de edad de los participantes se sitúa entre ocho y 10 años, dichas funciones no están plenamente adquiridas y el inicio del trabajo de estas puede resultar algo más complejo. Sin embargo, una vez familiarizados con las actividades e introducidas estas como rutina, puede afirmarse que la motivación e implicación de los alumnos se vio aumentada.

\section{Discusión y conclusiones}

A pesar de las controversias, sustentadas en la falta de consenso de los resultados obtenidos en los estudios precedentes, partimos del supuesto teórico que establece la relación entre la función ejecutiva y el rendimiento deportivo (Beavan, et al., 2020; Navarrete, 2019), para comprobar, en este estudio, si el rendimiento futbolístico de preadolescentes, correlaciona con el entrenamiento en las funciones ejecutivas. Los resultados obtenidos permiten concluir que existe una rela- 
ción positiva entre ambos constructos, de manera que cuanto mejor sea la función ejecutiva, mayor será el rendimiento en el fútbol.

Respecto al primero de los objetivos específicos, al evaluar la función ejecutiva en los participantes, se pudo comprobar, por un lado, coincidiendo con Alarcón et al. (2017a) que las capacidades cognitivas en los deportistas no son homogéneas, presentando una gran diversidad tanto en los niveles generales de FEs, como en las subdimensiones contempladas en la escala BRIEF-2. Más específicamente, los datos muestran que los participantes presentan ciertas FEs, que los sitúan en diferentes puntos, lo que podría ser fruto de su condición personal y de las influencias en otros contextos, de modo que la mayoría obtiene valores medios centrales y una leve minoría alcanza una elevada puntuación en FEs. Y por otro, tal y como señala Beavan et al. (2020), que las FEs no se mantienen constantes, sino que cambian conforme aumenta el tiempo de práctica deportiva, de manera que, cuanto mayor es el tiempo dedicado al fútbol, mejores serán los resultados obtenidos en las FEs, coincidiendo en este sentido, con los resultados obtenidos por Gómez et al. (2020) y San Miguel-Rodríguez (2020), entre otros. Así pues, se puede concluir que, aunque las FEs iniciales son relevantes, cuanto más tiempo se dedica a practicar fútbol, más inteligencia de juego futbolístico se adquiere, empleando la jerga lingüística de Beavan et al. (2019).

Del mismo modo, los resultados encontrados en este estudio, respecto a los niveles de rendimiento deportivo en fútbol que presentaron los participantes, son un claro reflejo de diversidad, quedando el talento y la excelencia reservada para una minoría. Ahora bien, considerando que los deportistas se hacen, y su construcción depende del dominio de los procesos cognitivos complejos (Palmi \& Riera, 2017), y dado que estas capacidades cognitivas, conocidas como ejecutivas, que explican su superioridad deportiva, podrían mejorarse fruto de la práctica repetida de actividades físicas en entornos con elevados niveles de entropía (Alarcón, et al., 2017b), se encuentra justificado que se incentiven en el futuro programas para promover esta capacidad a través del control de la tarea y el feedback del entrenador.

En referencia al segundo objetivo específico, al analizar si existe correlación entre el rendimiento del fútbol y la habilidad de la función ejecutiva, los resultados mostraron un nivel de rendimiento futbolístico, asociado al grado de dominio de las funciones ejecutivas una vez finalizada la aplicación del programa. Este hecho podría apuntar a que el entrenamiento en las FEs, du- rante las sesiones deportivas y fuera de ellas desde el hogar, contribuya al perfeccionamiento de las funciones ejecutivas, en línea con lo expuesto por Herrera (2012) quien en una investigación transversal ha encontrado la relación existente entre el rendimiento del fútbol sala y las funciones ejecutivas, y como los deportistas más expertos y con mayor práctica poseen valores superiores en estas capacidades en deportes, como fútbol, baloncesto y tenis. En base a todo ello, podemos afirmar que utilizar programas activos para trabajar las funciones ejecutivas en contextos similares al empleado en este estudio, sería una estrategia a contemplar para aumentar el rendimiento deportivo de los alumnos.

Concretamente, entre los hallazgos se encuentra el hecho de que los alumnos se habituaron a trabajar las FEs de forma sistemática, promoviendo su incorporación al repertorio conductual lo que podría incidir en el beneficio del adecuado manejo de las mismas en el rendimiento deportivo. Además, los niveles de concentración, motivación, satisfacción y rendimiento deportivos se incrementaron durante el transcurso del programa. Ahora bien, sería interesante poder comprobar, como una nueva línea de investigación ¿en qué medida, el grado de FEs y de rendimiento futbolístico perduran estables tras finalizar el tiempo de intervención? ¿Cuándo empiezan a decaer, si lo hacen, y a qué puede deberse? ¿Qué otros factores pueden condicionar el aumento de las FEs? Somos conscientes, porque así se ha expuesto en la fundamentación de este trabajo, que existen otros factores además del tiempo dedicado al deporte que pueden condicionar las FEs. Por tanto, se recomienda estudiar esta relación entre FEs y el rendimiento en el ejercicio del fútbol, ampliando y diversificando las variables tradicionales. De igual modo, se pueden establecer estudios descriptivos amplios que permitan conocer si existen diferencias en los niveles de FEs entre aquellos que practican deporte y aquellos que no, comprobando si existe disparidad en las habilidades cognitivas para la vida diaria, y si existen diferencias significativas en función de la modalidad deportiva que se práctica y del tiempo que se lleva en ella.

Por otra parte, aunque el instrumento cuantitativo empleado BRIEF-2 aporta una cobertura global y amplia de las FEs, y la observación participativa del entrenador permite obtener la información cualitativa, consideramos conveniente emplear otros instrumentos como los grupos de discusión, el autoseguimiento del propio deportista, a modo de ejemplo. En esta misma línea, se coincide con García et al. (2011) al considerar la necesidad de contar con un instrumento específico y 
adaptado que permita la evaluación de las FEs en el campo del deporte.

En cuanto a las limitaciones del estudio realizado, cabe señalar que la muestra empleada es reducida, no representativa, perteneciente a un solo club y exclusivamente al género masculino, lo que supone una amenaza para la posibilidad de generalizar los resultados obtenidos, e impide conocer las diferencias de género, quedando esto emplazado a futuros estudios. En lo que respecta al programa, la duración de las sesiones planteadas debería aumentarse, para que los resultados obtenidos fuesen más consistentes, y en futuros trabajos podría incluirse la variable rendimiento académico, para comprobar si las funciones ejecutivas están influyendo en actividades de tipo académico. Así mismo debería someterse a evaluación el papel que desempeña el entrenador, pues según Ordoqui (2020), no todos disponen de conocimientos rigurosos de la FEs y requieren de asesoramiento del psicólogo deportivo.

Para finalizar, señalar que si bien, en el presente trabajo se da una relación entre la práctica repetida de fútbol y el dominio de las funciones ejecutivas en los alumnos, es necesario contar con herramientas que identifiquen de forma concisa qué funciones ejecutivas son las que mejoran y cuáles son las herramientas para mejorarlas. Así mismo, y con base en los resultados de la revisión realizada por Paiano et al. (2019) aunque la mayoría de estudios asocian las actividades deportivas como el fútbol a la mejora de las funciones ejecutivas y habilidades atencionales en niños y adolescentes, y viceversa, las funciones ejecutivas mejoran el rendimiento deportivo, se considere todavía necesario seguir avanzando en la relación entre ambos constructos. Una buena praxis sería replicar los estudios para ver si se mantiene la hipótesis de forma constante, y en caso negativo, a qué puede ser debido.

\section{Referencias}

Alarcón, F., Ureña, N., Castillo, A., Martín, D. \& \& Cárdenas, D. (2017a). Las funciones ejecutivas como predictoras del nivel de pericia en jugadores de baloncesto. Revista de psicología del deporte, 26(1), 71-74.

American Psychology Association (2017). Ethical Principals of Psychologists and code of conduct.

Beavan, A., Spielmann, J., Mayer, J., Skorski, S., Meyer, T., \& Fransen, J. (2019). Agerelated differences in executive functions within high-level youth soccer players. Brazilian Journal of Motor Behavior, 13(2), 64 75. 10.20338/bjmb.v13i2.131
Beavan, A., Spielmann, J., Mayer, J., Skorski, S., Meyer, T., \& Fransen, J. (2020). The rise and fall of executive functions in high-level football players. Psychology of Sport and Exercise, 49, 152-166. https://doi.org/ 10.1016/j.psychsport.2020.101677

Bettega, O., Scaglia, A., Nascimento, J., Ibánez, S., \& Galatti, L. (2018). La enseñanza de táctica y técnica en el fútbol: conocimientos de entrenadores de categorías de formación. Retos. Nuevas tendencias en Educación Física, Deporte y Recreación, 33(1), 112-117. https://doi.org/10.47197/retos.v0i33.55503

Billat, V. (2002). Fisiología y metodología del entrenamiento de la teoría a la práctica. Editorial Paidotribo.

Cappuccio, M. (2017). Before Consciousness: In Search of the Fundamentals of Mind. Print Academy

Delis, D.C., Kaplan, E., \& Kramer, J.H. (2001). Sistema de funciones ejecutivas Delis-Kaplan (D-KEFS). PsycTests de la APA. https: / / doi.org/10.1037/t15082-000

Diamond, A. (2013). Executive functions. Annual Review of Psychology, 64, 135-168. https://doi.org/10.1146/ annurev-psych-113011-143750

Echavarría, L. (2017). Modelos explicativos de las funciones ejecutivas. Revista de Investigación en Psicología, 20(1), 237-247. https://doi.org/10.15381/ rinvp.v20i1.13367

García-Gómez, A. (2015). Desarrollo y validación de un cuestionario de observación para la evaluación de las funciones ejecutivas en la infancia. Revista Intercontinental de Psicología y Educación, 17(1), 141162.

García, S., Rodríguez, A., \& Garzón, A. (2011) Conceptualización de inteligencia táctica en el fútbol. Consideraciones para el desarrollo de un instrumento de evaluación en campo desde las funciones ejecutivas. Cuadernos de psicología del deporte, 11(1), 69-78.

Gómez, A., Cortina C., \& de la Garza Hinojosa, L.D. (2020). Memoria de trabajo y futbolistas femeniles: ¿Influye el tiempo de práctica? Trances:Transmisión del conocimiento educativo y de la salud, 12(5), 595-611. Recuperado de: https://revistatrances.wixsite.com/ trances/copia-de-12-5-3

González, G., Campos, M.C., \& Romero, S. (2014). Análisis de la influencia de la evaluación del rendimiento en jugadores de un equipo de fútbol. Retos: nuevas tendencias en educación física, deporte y recreación, 25, 85-89. https://doi.org/10.47197/ retos.v0i25.34486

Hernández-Mendo, A., Reigal, R. E., López-Walle, J. M., Serpa, S., Samdal, O., Morales-Sánchez, V., ... \& Falco, C. (2019). Physical activity, sports practice 
and cognitive functioning: The current research status. Frontiers in Psychology, 10, 2658.

Herrera, P. (7-8 de diciembre de 2011). Un microciclo de entrenamiento: modelo de construcción de la competición. I Congreso Internacional de fútbol. A Coruña. España. https: / /www.futbol-tactico.com/es/futbol/57/ confe-rencias / conferencia-paco-herrera-unmicrociclo-de-entrenamiento-modelo-deconstruccion-de-la-competicion.html

Macías, D., González-López, I., \& Eslava-Suanes, M.D. (2018). El fútbol como estrategias para el desarrollo de la inteligencia emocional del alumnado de Educación Primaria - Una experiencia didáctica. Educação \& Formação, Fortaleza, 3(8), 7-36. https: / / doi.org/ 10.25053 /redufor.v3i8.268

Merino, A., Arraiz, A., Sabiron, F., Usan, P. \& Jarie, L. (2017). La coordinación entre espacios formativos formales y deportivos: un estudio de caso etnográfico en el fútbol pre-benjamín. Ágora para la Educación Física y el Deporte, 19(2-3), 329-354. https: / / doi.org / 10.24197/aefd.2-3.2017.329-354

Moreno-Luque, M., Reigal Garrido, R. E., Morillo Baro, J. P., Morales Sánchez, V., \& Hernández Mendo, A. (2019). Estilo de interacción del entrenador, clima motivacional percibido y satisfacción de las necesidades psicológicas básicas en futbolistas jóvenes. Cuadernos De Psicología Del Deporte, 19(3), 79-89. https: / / doi.org/10.6018/cpd.372381

Navarrete, J. (2019). Rendimiento deportivo. Gaviota.

Ordoqui-Baldriche, J.A. (2020). Psicodiagnóstico y propuesta de intervención en el deporte de boxeo. Un estudio de caso. Revista Cubana de Medicina del Deporte y la Cultura Física, 9(1).

Organizacion Mundial de la Salud. (2017). La actividad física en los jovenes.

Paiano, R., Amaro, A. S., Garcia, F., Ferreira, R. C. T., Ressurreição, K. S., \& Carreiro, L. R. R. (2019). Futebol e funções executivas: um estudo de revisão. Cadernos de Pós-Graduação em Distúrbios do Desenvolvimento, 19(1), 81-97. https://dx.doi.org/ 10.5935/cadernosdisturbios.v19n1p81-97

Palmi, J., \& Riera, J. (2017). Las competencias del deportista para el rendimiento. Cuadernos de Psicología del Deporte, 17(1), 13-18.

Portellano, J.A., Martinez, A., \& Zumárraga, A. (2009). Evaluación de las Funciones Ejecutivas en niños. TEA Ediciones

Rivas-Martínez, H., \& Bailey-Moreno, J. (2019) Aportaciones del fútbol en la formación infantil y juvenil en una comunidad vulnerable. Retos 36, 459-468. https:/ /doi.org/10.47197/retos.v0i39.78491

Sanmiguel-Rodríguez, A. (2020). Grado de motivación y satisfacción de una plantilla de fútbol de la segunda división española. Retos, 40, 109-116. https: / / doi.org / 10.47197/retos.v1i40.79315

Santa-Cruz, C. \& Rosas, R. (2017). Cartografía de las Funciones Ejecutivas. Estudios de Psicología. 38(2), 295 305. https://doi.org/10.1080/ 02109395.2017.1311459

Serra, J., Prieto, A., Pastor, J. C., \& González, S. (2019). Proposal for a multidisciplinary assessment of talented football players. Retos, 38, 782-789. https: / /doi.org/10.47197/retos.v38i38.73118

Sousa, H., Gouveia, Élvio, Marques, A., Sarmento, H., Caldeira, R., Freitas, R., Lopes, H., Prudente, J., \& Ihle, A. (2021). The effect of balanced and unbalanced soccer small-sided games on the rating of perceived exertion in youth. Retos, 41, 440-446. https:// doi.org/10.47197/retos.v0i41.83091

Terry, C. (2006). The Martial Arts. Physical Medicine and Rehabilitation Clinics of NorthAmerica 17, 645676. https://doi.org/10.1016/j.pmr.2006.05.001

Vestberg T., Gustafson R., Maurex L., Ingvar M., \& Petrovic, P. (2012) Executive Functions Predict the Success of Top-Soccer Players. Plos one 7(4), e34731. https: / / doi.org/10.1371/journal.pone.0034731

Vickers, J.N. (2007). Entrenamiento de la percepción, la cognición y la toma de decisiones: el ojo tranquilo en acción. Cinética humana.

Webbe, F. (2011). The Handbook of Sport Neuropsychology. Springer Publishing Company.

Zuñiga Galaviz, U., Osorio Gutiérrez, A., Toledo Domínguez, I. de J., \& Herrera Perea, R. (2017). Somatotipo en futbolistas mexicanos profesionales de diferente nivel competitivo. Retos, 34, 100-102. https: / / doi.org/10.47197/retos.v0i34.52031

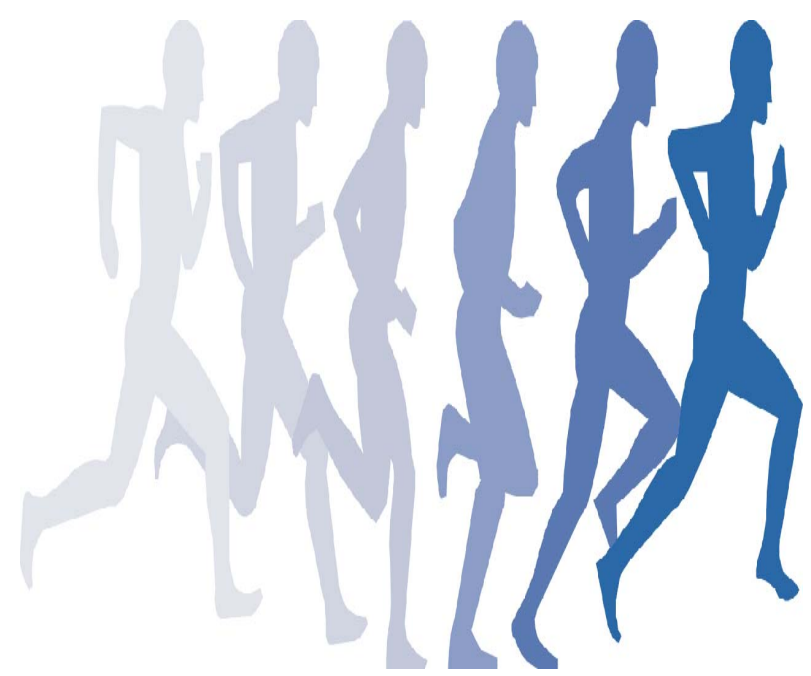

